\title{
Quantificadores Modulados e o Método da Semântica de Sociedades
}

\author{
Luiz Henrique C. Silvestrini
}

Departamento de Matemática, FC, UNESP 17033-360, Bauru, SP

E-mail: silvestrini@fc.unesp.br

\begin{abstract}
Resumo: As Semânticas de Sociedades, introduzidas por Carnielli e Lima-Marques (1999), estabelecem uma interpretação mais intuitiva para as inconsistências presentes em alguns sistemas formais. As sociedades foram formalizadas, originalmente, em duas classes principais, pois segundo o critério adotado para a aceitação de uma fórmula arbitrária $\varphi$, poderíamos ter sociedades abertas ou fechadas. Por exemplo, na sociedade fechada $S$, formada por um conjunto não vazio de agentes, $\varphi$ é aceita por $S$ somente no caso em que todos os agentes aceitam $\varphi$. Neste trabalho, propomos investigar o método da semântica de sociedades com relação aos quantificadores modulados, e mostrarmos as consequências de, mediante um arcabouço modelo-teorético, introduzir um quantificador modulado em uma semântica de sociedades.
\end{abstract}

Palavras-chave: Quantificadores Generalizados, Semântica de Sociedades .

\section{Introdução}

Carnielli e Lima-Marques [1] introduziram a Semântica de Sociedades, a qual se caracteriza como um tipo de construção lógica, que permite obter novas lógicas a partir da combinação dos agentes, i.e., das valorações de uma lógica estabelecida previamente. Esta abordagem situa-se numa área de estudo relativamente nova dentro da Lógica, a qual estuda combinações entre diferentes sistemas lógicos.

A partir das combinações de semânticas e lógicas não-clássicas, podemos resolver alguns problemas presentes no "estado da arte" das pesquisas em Lógica, tais como em Inteligência Artificial e Revisão de Crenças.

A motivação inicial desta proposta, deve-se ao estudo do processamento da informação obtida a partir de observações feitas por agentes "clássicos", mas onde o resultado de tal processo pudesse ter características não clássicas, tais como derrogar o princípio do terceiro excluído, pelas Sociedades Paracompletas em que não vale em geral $\varphi \vee \neg \varphi$; ou derrogar o princípio de explosão $\varphi, \neg \varphi \vdash \sigma$, em que contradição e trivialidade são noções distintas, assim como ocorre nas Sociedades Paraconsistentes.

Diante deste ambiente lógico, surge naturalmente a possibilidade de redefinir as sociedades abertas e fechadas por meio de quantificadores naturais, uma vez que as sociedades poderiam apresentar situações em que os quantificadores lógicos 'para todo' e 'existe' não 
seriam adequados para tal formalização. Dessa forma, buscamos investigar uma alternativa na formalização do método da Semântica de Sociedades.

O método da Semântica de Sociedades foi formalizado, originalmente, para alguns casos especiais de sociedades, a saber: as sociedades biassertivas, no sentido em que para cada variável proposicional $\varphi$, o valor lógico de $\neg \varphi$ independe do valor de $\varphi$.

Ademais, a semântica de sociedades foi formalizada para alguns casos particulares de sociedades, pois segundo o critério adotado para a aceitação das fórmulas, poderíamos ter, por exemplo, sociedades abertas ou fechadas, as quais são representadas, respectivamente, pelo sistema lógico paraconsistente $P^{l}$ (cf. [7]) e pelo sistema paracompleto $I^{l}$ (cf. [6]), ambos os sistemas são trivalentes. Apresentaremos, neste trabalho, as sociedades biassertivas fechadas, para então investigarmos o uso de quantificadores modulados a partir desta definição.

Um agente clássico, ou bivalorado, é uma valoração clássica $\alpha . \mathscr{L} \rightarrow\{0,1\}$; definida na linguagem $\mathscr{L}$ do Cálculo Proposicional Clássico (CPC). Uma sociedade de agentes clássicos é um conjunto não vazio $S$ de agentes clássicos.

Na sociedade fechada $S_{f}$, $\varphi$ é aceita por $S_{f}$ sempre que todos os agentes aceitam $\varphi$. Neste tipo de sociedade adota-se uma política fechada, no sentido em que $S_{f}$ aceita $\varphi$ se, e somente se, todos os agentes aceitam $\varphi$ e aceitará $\neg \varphi$ quando todos os agentes rejeitarem $\varphi$. Este tipo de sociedade é suscetível de não aceitar nem rejeitar $\varphi$, pois, por exemplo, podem existir agentes distintos $\alpha_{1}$ e $\alpha_{2}$ em que $\alpha_{1}$ aceita $\varphi$, entretanto, $\alpha_{2}$ rejeita $\varphi$ e, assim, a sociedade apresenta um caráter paracompleto e, ainda, a lei do terceiro excluído não é válida em geral. Nesse sentido, uma sociedade fechada teria um caráter intuicionista, visto que, por exemplo, $\varphi \vee \neg \varphi$ não é válida em geral.

Na sociedade aberta $S_{a}$, uma sentença $\varphi$ é aceita por $S_{a}$ sempre que existir, no mínimo, um agente que opine a favor de $\varphi$, e aceitará $\neg \varphi$ quando existir, no mínimo, um agente que rejeite $\varphi$. Uma sociedade deste tipo pode ser paraconsistente, pois $\varphi$ poderia ser aceita e rejeitada ao mesmo tempo por diferentes agentes.

Esta abordagem é análoga às ideias de S. Jaśkowski em sua proposta para a Lógica Discussiva, introduzida em 1948 (JAŚKOWSKI, 1969, cf. [4]), na qual há também uma aproximação às situações contraditórias a partir de opiniões que não são individualmente contraditórias.

Em 2003, Fernández e Coniglio (cf. [3]) propuseram uma generalização das Semânticas de Sociedades, por meio de um mecanismo de construção de novas lógicas pelo método de Semântica de Sociedades, e dessa forma, obtiveram uma hierarquia de lógicas paraconsistentes chamadas $\mathrm{P}^{n}$ (para $n \in \mathbb{N}$ ) e uma hierarquia de lógicas paracompletas denominadas $\mathrm{I}^{n}$ (para $n$ $\in \mathbb{N})$. 
Por outro lado, a partir do trabalho de Mostowski (1957, cf. [5]), evidenciou-se o fato de que existem muitos quantificadores, denominados quantificadores generalizados, que não são definíveis em termos daqueles usuais da lógica de primeira ordem clássica.

Carnielli e Grácio, em 2008 (cf. [2]) introduzem a família das lógicas moduladas, com particulares quantificadores generalizados, denominados quantificadores modulados, e estabelecem os respectivos modelos matemáticos.

Os sistemas lógicos modulados $\mathscr{L}_{\omega \omega}^{\tau}(\nabla)$ são caracterizados pela inclusão de um quantificador generalizado $\nabla$, ou seja, um quantificador que se encontra entre o universal $\forall$ e o existencial $\exists$, na sintaxe da lógica clássica de predicados de primeira ordem, cuja interpretação semântica é dada por um subconjunto do conjunto das partes do universo.

Os axiomas de $\mathscr{L}_{\omega \omega}^{\tau}(\nabla)$ são os da lógica clássica $\mathscr{L}_{\omega \omega}^{\tau}$, incluindo os axiomas da identidade, acrescentando-se alguns axiomas específicos para o quantificador $\nabla$, dentre eles destacamos: $\left(\mathrm{Ax}_{1}\right) \forall \mathrm{x} \theta(\mathrm{x}) \rightarrow \nabla \mathrm{x} \theta(\mathrm{x})$ e $\left(\mathrm{Ax}_{2}\right) \nabla \mathrm{x} \theta(\mathrm{x}) \rightarrow \exists \mathrm{x} \theta(\mathrm{x})$. As regras de inferência são Modus Ponens e Generalização.

A formalização de um sistema modulado permite obter inferências sobre objetos genéricos sobre um dado universo. Para isso, introduzimos uma nova regra de inferência definida em $\mathscr{L}_{\omega \omega}^{\tau}(\nabla)$, pela ampliação do tipo de similaridade por meio de uma nova constante $g$, denominada constante genérica. Assim, dado $\nabla \mathrm{x} \theta(\mathrm{x})$ inferimos, via regra do sistema modulado, $\theta(g)$.

Diante disso, neste trabalho apresentamos uma proposta de "sociedade modulada", em que a partir da estrutura de semântica de sociedade fechada, buscamos introduzir um quantificador modulado na mesma estrutura para definir quando um agente aceita ou rejeita $\varphi$.

\section{Materiais e Métodos}

Trata-se de um trabalho teórico e a presente pesquisa possibilita reconhecer o método das Semânticas de Sociedades em uma estrutura com quantificadores generalizados, dessa maneira, utilizaremos um tipo particular de sociedade fechada e um tipo particular de quantificador modulado.

\section{Objetivo}

Este trabalho tem como objetivo estabelecer critérios para uma investigação do método da semântica de sociedades com relação aos quantificadores modulados, e mostrarmos as consequências de, mediante um arcabouço modelo-teorético, introduzir um quantificador generalizado específico em um tipo de sociedade fechada. 


\section{Resultados e Discussão}

A semântica de sociedades foi originalmente formalizada por Carnielli e Lima-Marques para alguns casos especiais (cf. [1]). Contudo, podemos estabelecer outros critérios para decidir quando uma sociedade aceita $\varphi$. Para este objetivo, introduzimos os quantificadores modulados para reformular tal método e com isso desenvolvemos uma alternativa para a proposta introduzida por Carnielli e Lima-Marques.

Uma possibilidade de modular uma sociedade $S_{\mathrm{f}}$ seria adotar um quantificador de pseudo-topologia, por meio do seguinte critério: uma sociedade aceita $\varphi$ se, e somente se, boa parte dos agentes opine a favor de $\varphi$. Outro exemplo seria adotar o quantificador do ultrafiltro: uma sociedade aceita $\varphi$ se, e somente se, quase todos os agentes opinem a favor de $\varphi$.

Para o nível proposicional, buscamos estabelecer o critério abaixo para definir uma sociedade modulada.

Dada uma sociedade $S_{\mathrm{f}}=\left\{\alpha_{1}, \ldots, \alpha_{\mathrm{n}}, \ldots\right\}$ de agentes clássicos, definimos uma estrutura de sociedade modulada a partir de $S$, denotada por $m \nabla$, por meio da escolha de um quantificador modulado $\nabla$, adicionado na linguagem da sociedade $S_{\mathrm{f}}$, denotada por $\mathscr{L}(\nabla)$. Este quantificador modulado é interpretado por um conjunto de subconjuntos do conjunto das partes de $S_{\mathrm{f}}$, chamado complexo e denotado por $c$, i.e., $c \subseteq \wp\left(S_{\mathrm{f}}\right)-\{\varnothing\}$, assim tal estrutura é definida pelo seguinte par:

$$
m \nabla=\langle\mathfrak{J}, c\rangle
$$

em que, $\mathfrak{J}$ é considerada a estrutura subjacente à sociedade biassertiva gerada por $S_{\mathrm{f}}$.

A partir disso, podemos adaptar a definição de sociedade fechada pela inclusão de uma estrutura modulada, e por esta razão, tal sociedade é chamada de sociedade modulada, denotada por $\mathfrak{T}_{S}^{\nabla}$, se satisfaz as seguintes propriedades:

(i) $\mathfrak{J}_{S}^{\nabla}(\varphi)=1$ se, e somente se $\left\{\alpha \cdot \alpha \in S_{\mathrm{f}}, \alpha(\varphi)=1\right\} \in c$;

(ii) $\mathfrak{J}^{\nabla}{ }^{-}(\neg \varphi)=1$ se, e somente se $\left\{\alpha . \alpha \in S_{\mathrm{f}}, \alpha(\varphi)=0\right\} \in c$.

Assim, uma sociedade modulada aceita $\varphi$ se, e somente se, os indivíduos em $S_{\mathrm{f}}$ que aceitam $\varphi$ pertencem ao complexo $c$, ou seja, pertencem a um determinado conjunto definido por certas estruturas matemáticas (como a quase-topologia em [2] ou o ultrafiltro usual). Observamos que a escolha de um quantificador generalizado arbitrário, diferente dos explicitados aqui, pode gerar casos indiscerníveis, em que os agentes podem aceitar ou rejeitar qualquer sentença de modo trivial.

Para promover uma definição de Sociedade Modulada a partir de uma sociedade fechada de primeira ordem, consideramos os agentes como estruturas de primeira ordem. Dessa maneira, poderemos estender a relação de consequência fechada dada a partir de $\mathfrak{I}$. Neste caso, 
ao invés de exigirmos que todas as estruturas satisfaçam um predicado $P$, poderemos estabelecer, por exemplo, que boa parte das estruturas satisfaça tal predicado.

\section{Considerações Finais}

Neste trabalho, apresentamos uma investigação à teoria de semântica de sociedades combinada com quantificadores generalizados, de modo a formalizar tipos de sociedades por meio de quantificadores modulados, cujas estruturas são pseudo-topologia e ultrafiltro.

Contudo, apontamos para limitações ao se introduzir quantificadores generalizados, pois, dependendo da formalização do quantificador, os casos intermediários ao universal "para todo" e existencial "existe um", nas cláusulas definidoras para os agentes aceitarem ou rejeitarem alguma sentença $\varphi$, podem ficar todos indiscerníveis, ao menos do ponto de vista epistêmico.

\section{Referências}

[1] W. A. Carnielli; M. Lima-Marques. Society semantics for multiple-valued logics. In W. A. Carnielli and I.M.L. D'Ottaviano, editors, Advances in Contemporary Logic and Computer Science, volume 235 of Contemporary Mathematics Series, pp. 33-52. American Mathematical Society. 1999.

[2] W. A. Carnielli; M. C. C. Grácio. Modulated logic and flexible reasoning. Logic and Logical Philosophy, 17(3):211-249, 2008.

[3] V. L. Fernández; M. E. Coniglio. Combining valuations with society semantics. Journal of Applied Non-Classical Logics,13(1):21-46, 2003.

[4] S. JaŚkowski. Propositional calculus for contradictory deductive systems. Studia Logica, 24:143-157. Tradução para o inglês do artigo original em polonês publicado em 1948. 1969.

[5] A. Mostowski. On a generalization of quantifiers. Fundamenta Mathematicae, 44:12-36, 1957

[6] A. M. Sette; W. A. Carnielli. Maximal Weakly-Intuitionistic Logics. Studia Logica, v. 55, p. 181-203, 1995

[7] A. M. Sette. On the Propositional Calculus $\mathrm{P}^{1}$, Mathematica Japonicae, v. 18, p. 173-180, 1973 